\title{
Prognostic Value of Estrogen and Progesterone Receptor Expression in Low Proliferative Human Epidermal Growth Factor Receptor 2- Negative Breast Cancer
}

\author{
Yujin Lee, Inseok Park, Hyunjin Cho, Keunho Yang, Jungbin Kim, Kyeongmee Park ${ }^{1}$, Geumhee Gwak \\ Departments of Surgery and ${ }^{1}$ Pathology, Inje University Sanggye Paik Hospital, Inje University College of Medicine, Seoul, Korea
}

Purpose: Approximately two-thirds of breast cancer are estrogen-dependent cancers, which express estrogen receptor (ER)/progesterone receptor (PR). We investigated the prognostic value of ER/PR expression in human epidermal growth factor receptor 2 (HER2)-negative and low proliferative (Ki-67 $\leq 20 \%)$ breast cancer. Methods: A retrospective review was performed of 252 breast cancer data records, identified as ER/PR-positive, low Ki-67 proliferation index ( $\leq 20 \%)$ and HER2-negative. The data were divided into two subgroups: a strong luminal subgroup and a weak luminal subgroup, according to hormonal receptor expression status. Outcome measures included age at diagnosis, tumor size, tumor-node-metastasis (TNM) stage, ER, PR, Bcl-2, recurrent or metastatic characteristics, disease-free survival and overall survival, of each subgroup. Results: There were no statistical differences in TNM stage or tumor numbers between the two subgroups. The strong luminal subgroup was associated with a higher $\mathrm{Bcl}-2$ expression $(p<0.001)$. The weak luminal subgroup was associated with more frequent neural invasion ( $p=0.051)$ and lung $(p=0.031)$, liver $(p=0.031)$ and brain $(p=0.033)$ metastases, than the strong luminal subgroup. Disease-free survival was significantly longer in the strong luminal subgroup than weak luminal subgroup $(p=0.015)$. Overall survival was also significantly improved in the strong luminal subgroup relative to the weak luminal subgroup $(p=0.014)$. Conclusion: The weak luminal subgroup showed worse prognosis than the strong luminal subgroup, among ER/PR-positive HER2-negative low proliferative breast cancer patients. Weak ER or PR expression, can be considered a poor prognostic factor in ER/PR-positive HER2-negative low proliferative breast cancer.

Key Words: Breast neoplasms, Estrogen receptors, Progesterone receptors, Prognosis

\section{INTRODUCTION}

Breast cancer is the second leading cause of cancer death in women worldwide, although overall survival (OS) of breast cancer patients has been gradually increased over the last several decades, with advances in cancer therapeutics. Approximately two-thirds of breast cancer are estrogen-dependent cancers, which express estrogen receptor (ER)/ progesterone receptor (PR). It is well-established that anti-estrogen therapy, with tamoxifen or aromatase inhibitors (AIs), is effective to reduce recurrence and mortality rate of estrogen-dependent breast cancer. The National Surgical Adjuvant Breast and Bowel Project implemented the B-14 trial, the first randomized clinical trial to assess the efficacy of adjuvant tamoxifen treatment in patients with ER-positive breast cancer and negative axillary lymph nodes. At 5-year fol-

Correspondence: Geumhee Gwak

Department of Surgery, Inje University Sanggye Paik Hospital, Inje University

College of Medicine, 1342 Dongil-ro, Nowon-gu, Seoul 01757, Korea

Tel: +82-2-950-1017, Fax: +82-2-933-9389, E-mail: s2606@paik.ac.kr

Received: Aug 22, 2017 Revised: Oct 25, 2017 Accepted:Dec 5, 2017 low-up, tamoxifen treatment was found to have a highly statistically significant benefit in disease-free survival (DFS) of all ages [1,2]. Since the release of the first results of the Arimidex, Tamoxifen, Alone or in Combination (ATAC) trial, for adjuvant treatment of postmenopausal women with early breast cancer, AIs have widened the field of endocrine therapy in breast cancer and AIs show an enhanced antitumor effect compared to tamoxifen $[3,4]$. Despite a gradual decrease in death rate from breast cancer with anti-estrogen therapy, resistance to anti-estrogen therapy often develops in some estrogen-dependent tumors [5]. Since the first appearance of tamoxifen and AIs, endocrine-resistant breast cancer remains an active area of research $[6,7]$.

In our previous study, it was demonstrated that an ER/PR-decreased subgroup, among luminal B human epidermal growth factor receptor 2 (HER2)-negative breast cancer, was associated with a higher recurrence rate and shorter survival time than an ER/PR-intact subgroup [8]. The current study attempted to investigate the clinical implication of the decreased expression of ER/PR, in a more homogeneous breast cancer subgroup, identified as HER2-negative, low pro- 
liferative, with a Ki-67 below 20\%.

\section{METHODS}

Medical records were reviewed, of breast cancer patients who underwent breast surgery and treatment between September 2000 and October 2014 at Sanggye Paik Hospital. Two hundred ninety-seven breast cancer patients, with a low Ki-67 proliferation index $(\leq 20 \%)$ and HER2-negative from final pathologic reports, were identified. Data from 37 patients with stage 0 were excluded and triple-negative breast cancer was excluded, due to the small sample size $(n=8)$. The remaining 252 breast cancer data were divided into two subgroups: strong luminal and weak luminal, according to the strength of hormonal receptor expression. In both ER and PR immunohistochemical (IHC) staining, an Allred score of 7 or 8, was considered as belonging to the strong luminal subgroup and Allred scores between 2 and 6 were assigned to the weak luminal subgroup. Outcome measures included age at diagnosis, tumor size, node metastasis, breast and axilla operative methods, tumor type, tumor-node-metastasis (TNM) stage, histological grade, nuclear grade, tumor number, ER, PR, Ki-67 and bcl-2 expression, recurrent or metastatic characteristics, DFS and OS, of each subgroup. For the total recurrence rate, we compared the number of patients who were diagnosed as any site of metastasis. The patient with multiple metastasis was counted for each site of metastasis. The study was approved by the Institutional Review Board of Inje University Sanggye Paik Hospital (2017-01-012-002) and the informed consent was waived.

\section{IHC staining for ER, PR, Bcl-2, and $\mathrm{Ki}-67$}

The ER NCL-1-ER-6F11 and PR NCL-L-PGR-312 liquid mouse monoclonal antibodies (Leica Microsystems Inc., Newcastle Upon Tyne, UK), diluted 1:80 with normal goat serum (diluted 1:5 with Tris-buffered saline), were used as the primary antibodies for the ER and PR assays, respectively. The secondary antibody was goat anti-mouse peroxidase conjugated immunoglobulin. 3,3'-Diaminobenzidine tetrahydrochloride (DAB), was used as the chromogen. ER and PR were scored as $0,1+, 2+$, and $3+$, according to staining intensity, with a description of the percentage related to the proportion of stained nuclei in 10 high power fields [9]. The intensity scores and proportion of percentages were converted into Allred scores [10]. An
Allred score of 0 was defined as negative, 2 to 6 as weak positive and 7 or 8 as strong positive [11]. We defined Allred score over 6 of both ER and $\mathrm{PR}$ as strong positive.

IHC for Bcl-2 and $\mathrm{Ki}-67$ was performed using the avidin-biotin peroxidase complex method, with aminoethylcarbazole as the chromogen and the Vectastain ABC Elite kit (Vector Laboratories, Burlingame, USA). Bcl-2 overexpression was defined as Bcl-2 intensity above $33 \%$. The low proliferative group were identified, based on a Ki-67 cutoff at $20 \%$, which was described as the optimum for stratifying high-risk patients in luminal breast cancer [12].

\section{IHC staining for HER2/neu}

Binding of the primary antibody was detected using the Dako Quick-Staining, Labelled Streptavidin-Biotin System (Dako, Carpinteria, USA), followed by DAB chromogen addition. Each slide was scored in a blinded fashion by two pathologists, according to the manufacturer's recommended criteria. Intensity scores of 0 or $1+$ were designated as negative expression and 3+ was designated as a positive expression for HER2/neu. A 2+ score was considered equivocal and analyzed by silver-enhanced in situ hybridization (SISH) [9].

\section{SISH for HER2}

HER2 SISH was performed on a Ventana Benchmark automated instrument (Ventana Medical Systems Inc., Tucson, USA), according to the manufacturer's protocols for INFORM HER2 DNA and chromosome 17 probes. Evaluation of HER2 gene amplification status was performed in a blinded manner, using the American Society of Clinical Oncology/College of American Pathologists guidelines [13].

\section{Statistical methods}

The chi-square test was used to analyze the clinicopathological factors affecting the prognosis of the strong luminal subgroup and the weak luminal subgroup. The differences in DFS and OS between the two subgroups, were analyzed by the Kaplan-Meier method. The Cox multivariate regression model was used for the multivariate analysis. SPSS statistics version 21 (IBM Corp., Armonk, USA) and MedCalc statistical software version 17.6 (MedCalc Software, Ostend, Belgium), were used for the statistical analysis. $p<0.05$ was considered significant. 


\section{RESULTS}

Among a total of 252 breast cancer patients, 174 patients (69.0\%) were classified as belonging to the strong luminal subgroup and 78 patients (31.0\%) to the weak luminal subgroup.

\section{Patient characteristics}

There were no statistical differences in TNM stage or age at diagnosis (for a cutoff of 50 years) between the two subgroups. Both, mean and median age at diagnosis, were higher in the weak compared to strong luminal subgroup (52.3 vs. 51.3 and 50 vs. 48.5, respectively). The treatment modality including chemotherapy, hormone therapy, and radiotherapy did not show statistical differences between two subgroups (Table 1).

Table 1. Patient characteristics according to strength of ER and PR expression in low proliferative HER2 negative breast cancer

\begin{tabular}{|c|c|c|c|}
\hline & $\begin{array}{l}\text { Strong luminal } \\
\qquad \begin{array}{c}(n=174) \\
\text { No. }(\%)\end{array}\end{array}$ & $\begin{array}{l}\text { Weak luminal } \\
\qquad \begin{array}{c}(n=78) \\
\text { No. }(\%)\end{array}\end{array}$ & $p$-value \\
\hline Age (yr) & & & 0.587 \\
\hline Mean & 51.3 & 52.3 & \\
\hline Median & 48.5 & 50 & \\
\hline$\leq 50$ & $98(56.3)$ & $41(52.6)$ & \\
\hline$>50$ & $76(43.7)$ & $37(47.4)$ & \\
\hline TNM stage (AJCC 8th) & & & 0.347 \\
\hline I & $99(56.9)$ & $35(44.9)$ & \\
\hline ॥ & $62(35.6)$ & $35(44.9)$ & \\
\hline III & $12(6.9)$ & $7(8.9)$ & \\
\hline IV & $1(0.6)$ & $1(1.3)$ & \\
\hline Breast operation & & & 0.015 \\
\hline $\mathrm{BCS}$ & $138(79.3)$ & $50(64.1)$ & \\
\hline Mastectomy & $34(19.5)$ & $28(35.9)$ & \\
\hline None & $2(1.1)$ & 0 & \\
\hline Axillary node operation & & & 0.281 \\
\hline SLNB & $82(47.1)$ & $33(42.3)$ & \\
\hline ALND & $89(51.1)$ & 41 (52.6) & \\
\hline Omit & $3(1.7)$ & $4(5.1)$ & \\
\hline Chemotherapy & & & 0.123 \\
\hline Yes & $133(76.4)$ & $52(66.7)$ & \\
\hline No & $41(23.6)$ & $26(33.3)$ & \\
\hline Hormone therapy & & & 0.355 \\
\hline Yes & $163(93.7)$ & $76(97.4)$ & \\
\hline No & $11(6.3)$ & $2(2.6)$ & \\
\hline Radiotherapy & & & 0.887 \\
\hline Yes & $114(65.5)$ & $50(64.1)$ & \\
\hline No & $60(34.5)$ & $28(35.9)$ & \\
\hline
\end{tabular}

$E R=$ estrogen receptor; $P R=$ progesterone receptor; $H E R 2=$ human epidermal growth factor receptor 2; TMN = tumor-node-metastasis; $\mathrm{AJCC}=$ American Joint Committee on Cancer; BCS = breast-conserving surgery; SL$\mathrm{ND}=$ sentinel lymph node dissection; $\mathrm{ALND}=$ axillary lymph node dissection.

\section{Tumor characteristics}

There were no statistical differences in the tumor number, histologic type, histologic grade, nuclear grade, lymphatic invasion, vascular invasion and Ki-67 expression, between the two subgroups. However, the strong luminal subgroup was associated with a higher Bcl-2 expression $(>33 \%)(p<0.001)$ and the weak luminal subgroup was associated with more frequent neural invasion $(p=0.051)$ (Table 2$)$.

Table 2. Tumor characteristics according to strength of ER and PR expression in low proliferative HER2 negative breast cancer

\begin{tabular}{|c|c|c|c|}
\hline & $\begin{array}{l}\text { Strong luminal } \\
\qquad \begin{array}{c}(n=174) \\
\text { No. }(\%)\end{array}\end{array}$ & $\begin{array}{l}\text { Weak luminal } \\
\qquad(n=78) \\
\text { No. }(\%)\end{array}$ & $p$-value \\
\hline No. of tumors & & & 0.366 \\
\hline Single & $147(84.5)$ & $62(79.5)$ & \\
\hline Multiple & $27(15.5)$ & $16(20.5)$ & \\
\hline Histologic type & & & 0.293 \\
\hline Invasive ductal carcinoma & $143(82.2)$ & $67(85.9)$ & \\
\hline Invasive lobular carcinoma & $8(4.6)$ & $5(6.4)$ & \\
\hline Invasive mucinous carcinoma & $11(6.3)$ & $5(6.4)$ & \\
\hline Others* & $12(6.9)$ & $1(1.3)$ & \\
\hline Histologic grade & & & 0.2978 \\
\hline I & $32(18.4)$ & $15(19.2)$ & \\
\hline ॥ & $85(48.9)$ & $36(46.2)$ & \\
\hline III & $22(12.6)$ & $15(19.2)$ & \\
\hline Not available & $35(20)$. & $12(15.4)$ & \\
\hline Nuclear grade & & & 0.328 \\
\hline I & $12(6.9)$ & $10(12.8)$ & \\
\hline$\|$ & $106(60.9)$ & $44(56.4)$ & \\
\hline III & $21(12.1)$ & $12(15.4)$ & \\
\hline Not available & $35(20.1)$ & $12(15.4)$ & \\
\hline Lymphatic invasion & & & 0.161 \\
\hline No & $113(64.9)$ & $43(55.1)$ & \\
\hline Yes & $61(35.1)$ & $35(44.9)$ & \\
\hline Vascular invasion & & & 0.312 \\
\hline No & $161(96.0)$ & $71(91.0)$ & \\
\hline Yes & $7(4.0)$ & $6(7.7)$ & \\
\hline Not available & $6(13.2)$ & $1(1.3)$ & \\
\hline Neural invasion & & & 0.051 \\
\hline No & $136(78.2)$ & $50(64.1)$ & \\
\hline Yes & $15(8.6)$ & $9(11.5)$ & \\
\hline Not available & $23(13.2)$ & $19(24.4)$ & \\
\hline Bcl-2 expression (\%) & & & $<0.001$ \\
\hline$\leq 33$ & $11(6.3)$ & $19(24.4)$ & \\
\hline$>33$ & $163(93.7)$ & $59(75.6)$ & \\
\hline Ki-67 ( $\leq 20 \%)$ & & & 0.072 \\
\hline$\leq 14$ & $141(81.0)$ & $55(70.5)$ & \\
\hline$>14$ & $33(19.0)$ & $23(29.5)$ & \\
\hline
\end{tabular}

$E R=$ estrogen receptor; $\mathrm{PR}=$ progesterone receptor; $\mathrm{HER} 2=$ =human epidermal growth factor receptor 2 .

*Others include invasive medullary, tubular, papillary, cribriform, and micro-papillary carcinoma, apocrine cystic carcinoma, inflammatory breast cancer, mixed type breast cancer. 


\section{Recurrence sites}

Total recurrence rate, according to the strength of ER and PR expression, was higher in the weak luminal subgroup $(\mathrm{n}=18,23.1 \%)$ than the strong luminal subgroup $(\mathrm{n}=18,10.3 \%)(p<0.001)$. Distant

Table 3. Recurrence sites according to strength of ER and PR expression in low proliferative HER2 negative breast cancer

\begin{tabular}{lccc}
\hline & $\begin{array}{c}\text { Strong luminal } \\
(\mathrm{n}=174) \\
\text { No. }(\%)\end{array}$ & $\begin{array}{c}\text { Weak luminal } \\
(\mathrm{n}=78) \\
\text { No. }(\%)\end{array}$ & $p$-value \\
\hline $\begin{array}{c}\text { Breast } \\
\text { No }\end{array}$ & $164(94.3)$ & $72(92.3)$ & 0.582 \\
Yes & $10(5.7)$ & $6(7.7)$ & \\
Lymph node & & & 0.725 \\
No & $167(96.0)$ & $76(97.4)$ & \\
Yes & $7(4.0)$ & $2(2.6)$ & 0.076 \\
Bone & & & \\
No & $164(94.3)$ & $68(87.2)$ & 0.031 \\
Yes & $10(5.7)$ & $10(12.8)$ & \\
Lung & & & \\
No & $172(98.9)$ & $73(93.6)$ & 0.031 \\
Yes & $2(1.1)$ & $5(6.4)$ & \\
Liver & & & \\
No & $172(98.9)$ & $73(93.6)$ & 0.033 \\
Yes & $2(1.1)$ & $5(6.4)$ & \\
Brain & & & \\
No & $173(99.4)$ & $74(94.9)$ & \\
Yes & $1(0.6)$ & $4(5.1)$ & \\
Other visceral & $173(99.4)$ & $73(93.6)$ & \\
No & $1(0.6)$ & $5(6.4)$ & \\
Yes & & & \\
\hline
\end{tabular}

$E R=$ estrogen receptor; $\mathrm{PR}=$ progesterone receptor; $\mathrm{HER} 2$ = human epidermal growth factor receptor 2 .

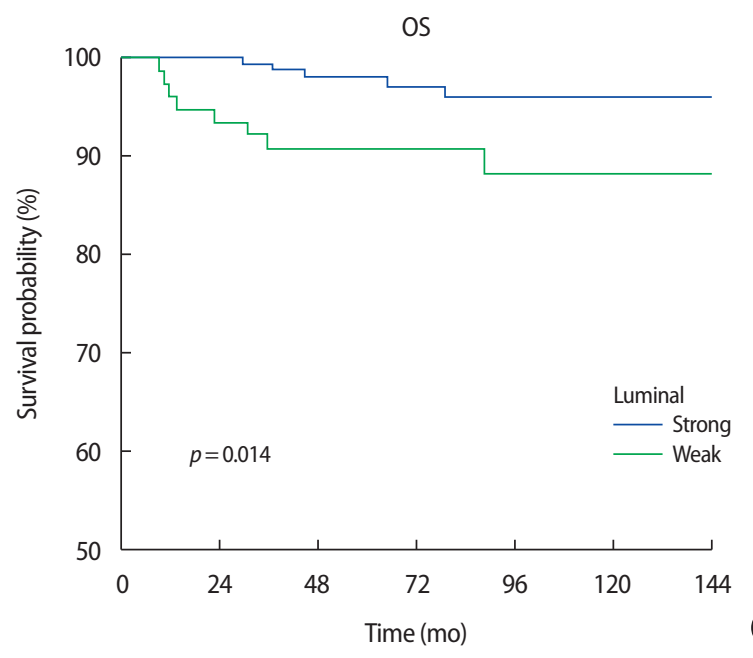

metastasis was also more frequently observed in the weak compared to strong luminal subgroup $(17.9 \%$ vs. $6.3 \%, p<0.001)$. A comparison of the recurrence rate, according to the recurrence sites: lung ( $p=$ $0.031)$, liver $(p=0.031)$, brain $(p=0.033)$ and other visceral organs $(p<0.001)$, showed metastasis occurred more frequently in the weak luminal subgroup than the strong luminal subgroup (Table 3). Among a total of 16 breast recurrence cases, 12 cases were ipsilateral and four cases were contralateral. Only one case of lymph node recurrence was contralateral among the total of nine cases. Total recurrence rate of the patient undergone modified radical mastectomy was significantly different between weak luminal subgroup (11/28, 39.3\%) and strong luminal subgroup $(3 / 34,8.8 \%)(p=0.006)$. Total recurrence rate of the patient undergone breast conserving surgery was higher in the weak luminal subgroup $(7 / 50,14 \%)$ than the strong luminal subgroup $(15 / 138,10.9 \%)$, but it was not significant statistically $(p=0.609)$.

\section{DFS and OS}

Both DFS and OS were significantly improved in the strong luminal subgroup relative to the weak luminal subgroup $(p=0.015$ and $p=0.014$, respectively) (Figure 1 )

\section{Multivariate analysis of prognostic factors}

In a multivariate model for OS, a relationship was confirmed for the strength of ER and PR expression, age, TNM stage, and histologic grade (Table 4). In this model, strong luminal group remained as an

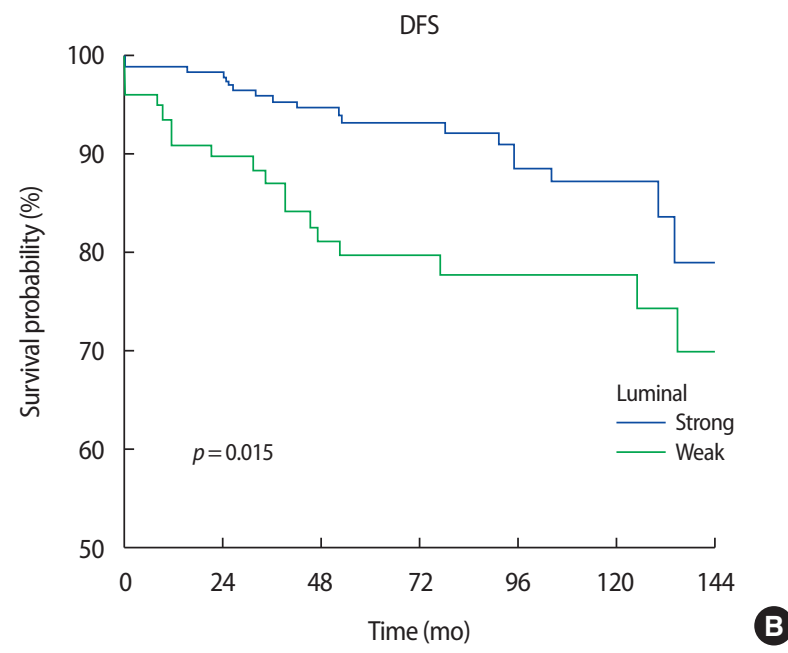

Figure 1. Overall survivals (OS) (A) and disease-free survivals (DFS) (B) according to the strength of estrogen receptor and progesterone receptor expression in low proliferative human epidermal growth factor receptor 2-negative breast cancer. 
Table 4. Multivariate analysis of factors associated with survival in low proliferative HER2 negative breast cancer

\begin{tabular}{lccr}
\hline Variable & Coefficient & $\mathrm{HR}(95 \% \mathrm{Cl})$ & $p$-value \\
\hline Strong luminal group & -1.252 & $0.286(0.089-0.914)$ & 0.035 \\
Stage III & 2.990 & $19.893(4.928-80.310)$ & $<0.001$ \\
Stage IV & 4.664 & $106.105(15.383-731.859)$ & $<0.001$ \\
Age & 0.060 & $1.061(1.008-1.118)$ & 0.024 \\
Histologic grade 3 & 1.040 & $2.829(0.823-9.725)$ & 0.099 \\
\hline
\end{tabular}

HER2 = human epidermal growth factor receptor $2 ; \mathrm{HR}=$ hazard ratio; $\mathrm{Cl}=$ confidence interval.

independent predictor of OS (strong luminal group versus weak luminal group: hazard ratio, 0.286 ; 95\% confidence interval, 0.089-0.914; $p=0.035)$.

\section{DISCUSSION}

For a patient with newly diagnosed breast cancer, the classification of the patient into either the high- or low-risk population of relapse and the need for adjuvant chemo-, radiation, anti-estrogen, or target therapy are controversial. Although node-negative breast cancer patients are considered as low-risk population for relapse, approximately up to $10 \%$ to $30 \%$ of these patients relapse within 10 years after initial therapy and the majority of recurrent breast cancer patients eventually die from the disease [14]. Accordingly, abundant clinical studies have been conducted, to identify the prognostic and predictive factors that enable clinicians to estimate the risk of relapse and the drug response of individual breast cancer patients. Risk assessment for relapse, is considered an essential factor in deciding the optimal therapeutic strategy and predicting the prognosis of breast cancer.

Over the last two decades, molecular profiling or multigene assays or IHC staining of ER, PR, HER2, Ki-67 and histologic grade, to identify at least four subtypes of breast cancer, such as luminal A, luminal B, HER2-enriched, and basal-like, have been investigated and performed, to improve the prognostication and risk stratification in breast cancer [15]. Ki-67, a nuclear marker of cell proliferation, is one of the most extensively studied markers in breast cancer [16]

A new cutoff value of Allred score 7 was defined for the strength of ER and PR expression in this research. High levels of ER expression (Allred scores of 7 and 8) ensured the response rate of neoadjuvant endocrine therapy for breast cancer patients in previous studies [17]. From this perspective, we investigated the significance of this value in determining the prognosis of ER/PR positive low proliferative HER2-negative breast cancer.

The Ki-67 cutoff value, to differentiate two luminal A and B categories, has been changing over time. The 2011 Saint Gallen Consensus Meeting, defined low-risk tumors as having a Ki-67 cutoff of $<14 \%$. In the 2013 Saint Gallen Conference, the majority of panelists voted that a threshold of $\geq 20 \%$ was indicative of high Ki- 67 status. For the last several years, many investigators proposed that a 20\% Ki-67 cutoff, is the best to stratify high-risk patients in luminal breast cancer [12]. In this context, the current study investigated the characteristics of low proliferative HER2-negative breast cancer patients, with a Ki67 cutoff of $20 \%$.

In many studies including meta-analyses, the expression of $\mathrm{Bcl}-2$ was confirmed as a favorable prognostic factor for breast cancer due to its correlation with ER status [18]. Recently, Dawson et al. [19] reported that $\mathrm{Bcl}-2$ is an independent parameter of a better prognosis for all types of early-stage breast cancer. In multivariable analysis, Ki67 was also concluded as an independent prognostic indicator, in terms of both DFS and OS for breast cancer [20]. When we analyzed tumor characteristics, according to the strength of ER and PR expression, the weak luminal subgroup was associated with frequent neural invasion, low Bcl-2 expression and a relatively higher Ki-67 (14\%-20\%) than the strong luminal subgroup. Therefore, the weak luminal subgroup is related to worse prognostic factors relative to the strong luminal subgroup.

Regarding distant metastatic recurrences and locoregional recurrences, we observed a more frequent distant metastasis, particularly to the lung, liver and brain, in the weak luminal subgroup than the strong luminal subgroup. We also observed a survival gap between the strong and weak luminal subgroups. These findings indicate that decreased ER/PR expression, has clinical prognostic implication in the HER2-negative luminal subgroup. Meaningfully, the weak luminal subgroup in this study, also includes cases with an Allred score of 5 and 6, expressing sufficient ER/PR to respond to endocrine therapy. This is the reason we carefully presume that decreased expression of ER/PR itself, might be related to drug resistance or metastatic potency.

The study by Balduzzi et al. [21] concluded tumors presenting low ER/PR expression, had similar outcomes when compared with triple-negative breast cancer. Their result showed no clear effect on survival outcomes with the addition of endocrine therapy, for patients 
with ER/PR expression of $1 \%$ to $10 \%$ [21]. The poor response to hormonal therapy, can be considered as a factor in explaining the worse prognosis of the weak compared to the strong luminal subgroup. In contrast, a meta-analysis of randomized trials concluded that even weakly positive ER, substantially benefited from tamoxifen but the proportional effect at a much higher ER was only slightly better [22].

Ono et al. [23] recently reported that PR expression and tumor size, were independent prognostic factors in the luminal A-like subtype and, also, the luminal A/PR-negative subtype had a higher risk of recurrence than the luminal A/PR-positive subtype. Given that the luminal A/PR-negative subtype largely overlapped with the weak luminal subgroup in our study, we carefully suggest that decreased ER/PR expression might be considered a meaningful prognostic factor in low proliferative HER2-negative breast cancer.

The weak luminal subgroup showed a worse prognosis than the strong luminal subgroup, among HER2-negative low proliferative breast cancer patients. Decreased expression of ER/PR itself, could be a clinical prognostic factor in HER2-negative low proliferative breast cancer. Current molecular classification of breast cancer represented as luminal type, is not sufficient for determining the high-risk group and additional gene analysis should be considered for the patients with relatively poor prognostic factors, like low ER/PR expression.

\section{CONFLICT OF INTEREST}

The authors declare that they have no competing interests.

\section{REFERENCES}

1. Fisher B, Costantino J, Redmond C, Poisson R, Bowman D, Couture J, et al. A randomized clinical trial evaluating tamoxifen in the treatment of patients with node-negative breast cancer who have estrogen-receptor-positive tumors. N Engl J Med 1989;320:479-84.

2. Fisher B, Redmond C. Systemic therapy in node-negative patients: updated findings from NSABP clinical trials. National Surgical Adjuvant Breast and Bowel Project. J Natl Cancer Inst Monogr 1992;(11):105-16.

3. Baum M, Budzar AU, Cuzick J, Forbes J, Houghton JH, Klijn JG, et al. Anastrozole alone or in combination with tamoxifen versus tamoxifen alone for adjuvant treatment of postmenopausal women with early breast cancer: first results of the ATAC randomised trial. Lancet 2002;359:2131-9.

4. Cuzick J, Sestak I, Baum M, Buzdar A, Howell A, Dowsett M, et al. Effect of anastrozole and tamoxifen as adjuvant treatment for early-stage breast cancer: 10-year analysis of the ATAC trial. Lancet Oncol 2010;11:1135-41.

5. Musgrove EA, Sutherland RL. Biological determinants of endocrine resistance in breast cancer. Nat Rev Cancer 2009;9:631-43.

6. Lauring J, Wolff AC. Evolving role of the estrogen receptor as a predictive biomarker: ESR1 mutational status and endocrine resistance in breast cancer. J Clin Oncol 2016;34:2950-2.

7.Zhang M, Chen H, Gu J. Analysis of factors affecting endocrine therapy resistance in breast cancer. Oncol Lett 2016;11:379-84.

8. Park C, Park K, Kim J, Sin Y, Park I, Cho H, et al. Prognostic values of negative estrogen or progesterone receptor expression in patients with luminal B HER2-negative breast cancer. World J Surg Oncol 2016;14:244.

9. Kim HS, Park I, Cho HJ, Gwak G, Yang K, Bae BN, et al. Analysis of the potent prognostic factors in luminal-type breast cancer. J Breast Cancer 2012;15:401-6.

10. Allred DC, Harvey JM, Berardo M, Clark GM. Prognostic and predictive factors in breast cancer by immunohistochemical analysis. Mod Pathol 1998;11:155-68.

11. Phillips T, Murray G, Wakamiya K, Askaa J, Huang D, Welcher R, et al. Development of standard estrogen and progesterone receptor immunohistochemical assays for selection of patients for antihormonal therapy. Appl Immunohistochem Mol Morphol 2007;15: 325-31.

12. Bustreo S, Osella-Abate S, Cassoni P, Donadio M, Airoldi M, Pedani F, et al. Optimal Ki67 cut-off for luminal breast cancer prognostic evaluation: a large case series study with a long-term follow-up. Breast Cancer Res Treat 2016;157:363-71.

13. Park K, Han S, Kim JY, Kim HJ, Kwon JE, Gwak G. Silver-enhanced in situ hybridization as an alternative to fluorescence in situ hybridization for assaying HER2 amplification in clinical breast cancer. J Breast Cancer 2011;14:276-82.

14. Fisher B, Slack NH, Bross ID. Cancer of the breast: size of neoplasm and prognosis. Cancer 1969;24:1071-80.

15. Braunstein LZ, Taghian AG. Molecular phenotype, multigene assays, and the locoregional management of breast cancer. Semin 
Radiat Oncol 2016;26:9-16.

16. Honma N, Horii R, Iwase T, Saji S, Younes M, Ito Y, et al. Ki-67 evaluation at the hottest spot predicts clinical outcome of patients with hormone receptor-positive/HER2-negative breast cancer treated with adjuvant tamoxifen monotherapy. Breast Cancer 2015;22:71-8.

17. Ellis MJ, Rosen E, Dressman H, Marks J. Neoadjuvant comparisons of aromatase inhibitors and tamoxifen: pretreatment determinants of response and on-treatment effect. J Steroid Biochem Mol Biol 2003;86:301-7.

18. Callagy GM, Webber MJ, Pharoah PD, Caldas C. Meta-analysis confirms BCL2 is an independent prognostic marker in breast cancer. BMC Cancer 2008;8:153.

19. Dawson SJ, Makretsov N, Blows FM, Driver KE, Provenzano E, Le Quesne J, et al. BCL2 in breast cancer: a favourable prognostic marker across molecular subtypes and independent of adjuvant therapy received. Br J Cancer 2010;103:668-75.

20. Inwald EC, Klinkhammer-Schalke M, Hofstädter F, Zeman F,
Koller M, Gerstenhauer M, et al. Ki-67 is a prognostic parameter in breast cancer patients: results of a large population-based cohort of a cancer registry. Breast Cancer Res Treat 2013;139:539-52.

21. Balduzzi A, Bagnardi V, Rotmensz N, Dellapasqua S, Montagna E, Cardillo A, et al. Survival outcomes in breast cancer patients with low estrogen/progesterone receptor expression. Clin Breast Cancer 2014;14:258-64.

22. Early Breast Cancer Trialists' Collaborative Group (EBCTCG), Davies C, Godwin J, Gray R, Clarke M, Cutter D, et al. Relevance of breast cancer hormone receptors and other factors to the efficacy of adjuvant tamoxifen: patient-level meta-analysis of randomised trials. Lancet 2011;378:771-84.

23. Ono M, Tsuda H, Yoshida M, Shimizu C, Kinoshita T, Tamura K. Prognostic significance of progesterone receptor expression in estrogen-receptor positive, HER2-negative, node-negative invasive breast cancer with a low Ki-67 labeling index. Clin Breast Cancer 2017;17:41-7. 